\title{
Chicken-Wire Vasculature
}

National Cancer Institute

\section{Source}

National Cancer Institute. Chicken-Wire Vasculature. NCI Thesaurus. Code C96347.

A morphologic finding that refers to delicate vascular channel formations. It is characterized by the formation of arborizing and curving capillaries. 